\title{
Utility Representation of Lower Separable Preferences
}

\author{
Özgür Yılmaz*
}

June 2008

\begin{abstract}
Topological separability is crucial for the utility representation of a complete preference relation. When preferences are incomplete, this axiom has suitably defined counterparts: upper separability and lower separability (Ok (2002)). We consider the problem of representing an incomplete preference relation by means of a vector-valued utility function; we obtain representation results under the lower separability assumption. Our results extend the main representation theorems by Ok (2002) in terms of the separability axioms.
\end{abstract}

Keywords: Incomplete Preferences, Utility Representation, Upper/Lower Separability, Near-completeness

Journal of Economic Literature Classification Number: D11

\section{Introduction}

Preference relations are usually assumed to satisfy completeness and transitivity. These two properties have been the core axioms, tying self-interest to 'rationality'. They are also useful for analytical tractability. Particularly, the possibility of representation by real-valued functions owes much to the completeness axiom. However, for most problems of individual decision making, it seems unrealistic to assume completeness. Our focus is the utility representation without the completeness axiom.

${ }^{*}$ College of Administrative Science and Economics, Koç University, Sarıyer, İstanbul, Turkey 34450; Fax:+90 212338 1653; E-mail address: ozyilmaz@ku.edu.tr 
Obviously, an incomplete preorder can not be represented by a real-valued utility function. The scope of this limitation of analytical tractability is extensively discussed in the literature. ${ }^{1}$ Basically, in the case of incomplete preferences, while utility representation is not possible, one may still find a utility function that represents the preference order in a weaker sense: as before, if $x$ is preferred to $y$ then $u(x)>u(y)$, but the opposite implication is no longer true. This approach is explored by Richter (1966) and Peleg (1970). Clearly, this 'quasi-representation' is very much problematic, because the preorder cannot be recovered from the utility function $u$.

An alternative approach is representation by means of a vector-valued utility function. ${ }^{2}$ The question is whether, given a preorder $\succsim$, it is possible to find a function $\mathbf{u}: X \rightarrow \mathbb{R}^{n}$ for some positive integer $n$ such that, for each $x, y \in X$,

$$
x \succsim y \text { if and only if } \mathbf{u}(x) \geq \mathbf{u}(y) .
$$

As a natural extension of the classical utility representation, multi-criteria decision making is not only intuitive but also analytically convenient. Ok (2002) shows that, under certain topological separability assumptions, a not-too-incomplete preference relation, a preference relation such that any subset of mutually incomparable alternatives is finite, can be represented by a vector-valued utility function. First, he suggests two counterparts of the usual separability axiom, upper separability and lower separability, which are suitably defined for incomplete preferences. Then, he shows that upper separability is sufficient for the vector-valued utility representation; also, he shows that upper and lower separability together imply the existence of an upper semicontinuous vector-valued utility representation. We extend these results in terms of the separability axioms: we show that lower separability is sufficient for representability by means of a vector-valued utility function (Theorem 3); it follows from this result that lower separability is also the key axiom for the existence of such a function that is upper semicontinuous (Theorem 4).

\footnotetext{
${ }^{1}$ See Aumann (1962), Richter (1966), Peleg (1970), and Sondermann (1980).

${ }^{2}$ For a discussion of the multi-attribute utility representation, see Ok (2002) and Eliaz and Ok (2006).
} 


\section{Preliminary Definitions and Existing Results}

Let $\succcurlyeq$ be a binary relation on a set of alternatives $X$. Two alternatives $x$ and $y$ are $\succcurlyeq$-comparable if either $x \succcurlyeq y$ or $y \succcurlyeq x$ holds. They are $\succcurlyeq$-incomparable if they are not $\succcurlyeq$-comparable. For each $\succcurlyeq$-incomparable pair of alternatives $x$ and $y$, we write $x \bowtie y$. A binary relation $\succcurlyeq$ is complete if and only if each $x$ and $y$ in $X$ are $\succcurlyeq$-comparable. The strict (asymmetric) part of $\succcurlyeq$, denoted by $\succ$, is the relation on $X$ defined as $x \succ y$ if and only if $x \succcurlyeq y$ and $\neg(y \succcurlyeq x)$. The symmetric part of $\succcurlyeq$, denoted by $\sim$, is the relation on $X$ defined as $x \sim y$ if and only if $x \succcurlyeq y$ and $y \succcurlyeq x$. For each $Y \subseteq X$, the relation induced by $\succcurlyeq$ on $Y$, denoted $\left.\succcurlyeq\right|_{Y}$, is defined as $\left.\succcurlyeq\right|_{Y} \equiv \succcurlyeq \cap(Y \times Y)$. The relation $\succcurlyeq^{\prime}$ is an extension of $\succcurlyeq$, if $\succcurlyeq \subseteq \succcurlyeq^{\prime}$ and $\succ \subseteq \succ^{\prime}$. A relation $\succcurlyeq$ is a preorder if $\succcurlyeq$ is reflexive and transitive, a partial order if it is an antisymmetric preorder, a linear order if it is a complete partial order.

A preordered set is a pair $(X, \succcurlyeq)$ such that $X$ is a nonempty set and $\succcurlyeq$ is a preorder on $X$. Let $(X, \succcurlyeq)$ be a preordered set. A subset $Y$ of $X$ is $\succcurlyeq$-dense if, for each pair $x, y \in X$ with $x \succ y$, there is $z \in Y$ such that $x \succ z \succ y$. The preorder $\succcurlyeq$ is weakly separable if there is a countable $\succcurlyeq$-dense set in $X$. Clearly, if $\succcurlyeq$ is weakly separable, then $X$ is infinite.

A preordered set $(X, \succcurlyeq)$ is a poset (partially ordered set) if $\succcurlyeq$ is a partial order on $X$. Given a poset $(X, \succcurlyeq)$, the partial order $\succcurlyeq$ is spacious if $x \succ y$ implies that $\{z: z \in X$ and $x \succ z\} \supseteq$ Closure $\{z: z \in X$ and $y \succ z\}$.

Theorem 1 Peleg (1970) Let $\succcurlyeq$ be a partial order on a nonempty set $X$. If for each $x \in X$, $\{y: y \in X$ and $x \succ y\}$ is open and $\succcurlyeq i s$ weakly separable and spacious, then there exists a continuous function $u: X \rightarrow[0,1]$ such that for each $x, y \in X, x \succ y$ implies $u(x)>u(y)$.

A poset $(X, \succcurlyeq)$ is a chain if $\succcurlyeq$ is a linear order, and an antichain if $\succ=\emptyset$. Clearly, if $(X, \succcurlyeq)$ is a chain, then $\bowtie=\emptyset$, and if it is an antichain, then $\bowtie=\{(x, y): x \neq y\}$. A chain in a poset $(X, \succcurlyeq)$ is a set $Y \subseteq X$ such that the relation $\left.\succcurlyeq\right|_{Y}$ is complete. Similarly, a set $Y \subseteq X$ is an antichain in a poset $(X, \succcurlyeq)$ if $\left.\succ\right|_{Y}=\emptyset$. An antichain $Y$ in $(X, \succcurlyeq)$ is of maximal cardinality if its cardinality is at least as large as that of each antichain in $(X, \succcurlyeq)$. In this case, the cardinality of $Y$ is the width of the poset $(X, \succcurlyeq)$. It is denoted by $w(X, \succcurlyeq)$. Clearly, the width of each chain 
is zero, while the width of an antichain $(X, \succcurlyeq)$ is $|X|$. An important result which we will use extensively in the following analysis is Dilworth's Decomposition Theorem (1950): If $(X, \succcurlyeq)$ is a poset with finite width $w$, then $X$ is the union of $w$ chains in $(X, \succcurlyeq)$. For each poset $(X, \succcurlyeq)$, let $\mathcal{L}(X, \succcurlyeq)$ be the set of all extensions of $\succcurlyeq$ that are linear orders. By the classical Szpilrajn's Theorem (1930), each partial order can be extended to a linear order. Thus, for each poset $(X, \succcurlyeq), \mathcal{L}(X, \succcurlyeq) \neq \emptyset$. From this result, it follows that, for each poset $(X, \succcurlyeq)$,

$$
\succcurlyeq=\underset{R \in \mathcal{L}(X, \succcurlyeq)}{R,}
$$

that is, each partial order is the intersection of all of its linear extensions. The order dimension of a poset $(X, \succcurlyeq)$, denoted as $\operatorname{dim}(X, \succcurlyeq)$, is the minimum number of linear extensions of $\succcurlyeq$, the intersection of which is $\succcurlyeq$, provided that this number is finite, and is $\infty$, otherwise. That is,

$$
\operatorname{dim}(X, \succcurlyeq) \equiv \min \left\{k \in \mathbb{N}: R_{i} \in \mathcal{L}(X, \succcurlyeq), i=1, \ldots, k, \text { and } \succcurlyeq=\underset{i=1}{n} R_{i}\right\}
$$

For each poset $(X, \succcurlyeq), w(X, \succcurlyeq) \geq \operatorname{dim}(X, \succcurlyeq)$ (Hiragushi (1955)).

A partial order $\succcurlyeq$ is representable if there is a positive integer $n$ and a function $\mathbf{u}: X \rightarrow \mathbb{R}^{n}$ such that, for each $x, y \in X$,

$$
x \succcurlyeq y \Leftrightarrow \mathbf{u}(x) \geq \mathbf{u}(y) .
$$

Proposition 1 Ok (2002) Let $\succcurlyeq$ be a partial order on a nonempty set $X$. If $\succcurlyeq$ is representable, then $\operatorname{dim}(X, \succcurlyeq)<\infty$. Moreover, if $X$ is countable and $\operatorname{dim}(X, \succcurlyeq)<\infty$, then $\succcurlyeq$ is representable.

Since $w(X, \succcurlyeq) \geq \operatorname{dim}(X, \succcurlyeq)$, it implies, that if $X$ is countable and $w(X, \succcurlyeq)$ is finite, then $\succcurlyeq$ is representable. Our focus, on the other hand, is on representability without the restriction that $X$ is a countable set. We analyze this problem in the following section.

\section{Representation of Near-Complete Preferences}

A natural conception of the 'degree of incompleteness' is near-completeness: A preorder is nearcomplete, if each subset $A$ of $X$, with $x \bowtie y$ for each distinct $x, y$ in $A$, is finite (Ok (2002)). 
Thus, a partial order $\succcurlyeq$ on $X$ is near-complete if and only if $w(X, \succcurlyeq)<\infty$.

Let $(X, \succcurlyeq)$ be any preordered set. A subset $Y$ of $X$ is upper $\succcurlyeq$-dense if, for each $x, y \in X$ such that $x \bowtie y$, there is $z \in Y$ such that $x \succ z \bowtie y$. It is lower $\succcurlyeq$-dense if, for each $x, y \in X$ such that $x \bowtie y$, there is $z \in Y$ such that $x \bowtie z \succ y$. A preorder $\succcurlyeq$ is upper (lower) separable if there is a countable set in $X$ which is both $\succcurlyeq$-dense and upper (lower) $\succcurlyeq$-dense. Finally, $\succcurlyeq$ is separable if it is both upper and lower separable.

Theorem 2 Ok (2002) Let $X$ be any nonempty set and let $\succcurlyeq$ be a near-complete and upper separable partial order on $X$. Then, $\succcurlyeq$ is representable.

Our first result is the counterpart of this theorem in terms of the separability assumption. We replace upper separability with lower separability and obtain a representability result.

Theorem 3 Let $X$ be any nonempty set and let $\succcurlyeq$ be a near-complete and lower separable partial order on $X$. Then, $\succcurlyeq$ is representable.

Proof. Let $(X, \succcurlyeq)$ be a poset such that its width is $n<\infty$, and $\succcurlyeq$ is lower separable. By Dilworth's Decomposition Theorem, there is a partition of $X$, say $X_{1}, X_{2}, \ldots, X_{n}$ such that, for each $k,\left.\succcurlyeq\right|_{X_{k}}$ is a linear order on $X_{k}$. For each $k$, define $R_{k}$ and $R_{k}^{\prime}$ as follows:

$$
\begin{aligned}
& x R_{k} y \quad \Leftrightarrow x \notin X_{k} \text { and } \exists z \in X_{k} \text { s.t. } x \succ z \bowtie y, \\
& x R_{k}^{\prime} y \quad \Leftrightarrow \quad x \in X_{k} \text { and } x \bowtie y .
\end{aligned}
$$

Then define the relation $\unrhd_{k}=\succcurlyeq \cup R_{k} \cup R_{k}^{\prime}$.

Step 1: $\unrhd_{k}$ is a partial order.

- Reflexivity of $\unrhd_{k}$ : It follows from reflexivity of $\succcurlyeq$.

- Antisymmetry of $\unrhd_{k}$ : Let $x \unrhd_{k} y \unrhd_{k} x$. Suppose $x \neq y$. There are three cases: i) $x \succ y$.

Thus, $\neg(y \bowtie x)$, and it implies $\neg\left(y R_{k}^{\prime} x\right)$. Since $\succcurlyeq$ is antisymmetric, $y R_{k} x$. By definition of $R_{k}$, there is $z \in X_{k}$ such that $y \succ z \bowtie x$. Since $x \succ y$, transitivity of $\succcurlyeq$ implies that $x \succ z$. It contradicts with $z \bowtie x$. 
ii) $x R_{k} y$.

By definition of $R_{k}, x \notin X_{k}$ and there is $z \in X_{k}$ such that $x \succ z \bowtie y$. Suppose $y R_{k} x$. Then, there is $z^{\prime} \in X_{k}$ such that $x \succ z \bowtie y \succ z^{\prime} \bowtie x$. Clearly, $z=z^{\prime}$ is impossible. If $z \succ z^{\prime}$, then transitivity of $\succcurlyeq$ implies $x \succ z^{\prime}$. It contradicts with $z^{\prime} \bowtie x$. If $z^{\prime} \succ z$, transitivity of $\succcurlyeq$ implies $y \succ z$. It contradicts with $z \bowtie y$. Note that since $z$ and $z^{\prime}$ are in $X_{k}$, and $X_{k}$ is a chain, $z$ and $z^{\prime}$ are $\succcurlyeq$-comparable. Suppose $y \succ x$. It implies $x \succ z \bowtie y \succ x$. This contradicts with transitivity of $\succcurlyeq$. Finally, since $z \in X_{k}, y \notin X_{k}$ and $\neg\left(y R_{k}^{\prime} x\right)$.

iii) $x R_{k}^{\prime} y$.

By definition of $R_{k}^{\prime}, x \in X_{k}$ and $x \bowtie y$. Since $X_{k}$ is a chain, $y \notin X_{k}$ and $\neg\left(y R_{k}^{\prime} x\right)$. Thus, $y R_{k} x$, and there is $z \in X_{k}$ such that $y \succ z \bowtie x$. But, since $x, z \in X_{k}$, it is not possible. Thus, $x \unrhd_{k} y \unrhd_{k} x$ implies $x=y$. Thus, $\unrhd_{k}$ is antisymmetric.

- Transitivity of $\unrhd_{k}$ : Let $x \unrhd_{k} y \unrhd_{k} z$. If $x \succcurlyeq y \succcurlyeq z$, then, by transitivity of $\succcurlyeq, x \succcurlyeq z$. Note that, $x R_{k}^{\prime} y$ implies $y \notin X_{k}$ and $\neg\left(y R_{k}^{\prime} z\right)$ for each $z \in X$. Thus, $x R_{k}^{\prime} y R_{k}^{\prime} z$ is not possible. Also, since $X_{k}$ is a chain and $x \succ a \bowtie y$ for some $a \in X_{k}, y \notin X_{k}$. Then, for each $z \in X, \neg\left(y R_{k}^{\prime} z\right)$. Thus, $x R_{k} y R_{k}^{\prime} z$ is not possible. There are six cases left to be considered:

i) $x \succcurlyeq y R_{k} z$

By definition of $R_{k}, y \notin X_{k}$ and there is $a \in X_{k}$ such that $x \succcurlyeq y \succ a \bowtie z$. If $x \notin X_{k}$, then transitivity of $\succcurlyeq$ implies $x R_{k} z$. Suppose $x \in X_{k}$. Since $x \succcurlyeq y \succ a \bowtie z$, either $x \succcurlyeq z$ or $x \bowtie z$. If $x \bowtie z$, then $x R_{k}^{\prime} z$. In either case, $x \unrhd_{k} z$.

ii) $x R_{k} y \succcurlyeq z$

There is $a \in X_{k}$ such that $x \succ a \bowtie y \succcurlyeq z$, where $x \notin X_{k}$. Since $a$ and $y$ are $\succcurlyeq$-incomparable, either $a \succ z$ or $a \bowtie z$. If $a \succ z$, then $x \succ z$. If $a \bowtie z$, then, since $x \notin X_{k}$ and $a \in X_{k}, x \succ a \bowtie z$ implies $x R_{k} z$. Thus, $x \unrhd_{k} z$.

iii) $x R_{k}$ y $R_{k} z$

There are $a, b \in X_{k}$ such that $x \succ a \bowtie y \succ b \bowtie z$ where $x, y \notin X_{k}$. Since $X_{k}$ is a chain, $a$ and $b$ are $\succcurlyeq$-comparable. Since $x \succ a \bowtie y \succ b, a \neq b$. If $b \succ a$, then $y \succ a$. It is a contradiction. Thus, $a \succ b$. By transitivity of $\succcurlyeq, x \succ b \bowtie z$. Thus, $x \unrhd_{k} z$.

iv) $x \succcurlyeq y R_{k}^{\prime} z$ 
If $x \notin X_{k}$, then, since $y \in X_{k}$ and $x \succ y \bowtie z, x R_{k} z$. Suppose $x \in X_{k}$. If $z \succcurlyeq x$, then $z \succcurlyeq y$. It is a contradiction. Thus, either $x \bowtie z$ or $x \succ z$. If $x \bowtie z$, then, by definition of $R_{k}^{\prime}, x R_{k}^{\prime} z$. In both cases, $x \unrhd_{k} z$.

v) $x R_{k}^{\prime} y \succcurlyeq z$

Clearly, $z \succcurlyeq x$ is not possible. Thus, either $x \succcurlyeq z$ or $x \bowtie z$. Since $x \in X_{k}$, the latter implies $x R_{k}^{\prime} z$. Thus, $x \unrhd_{k} z$.

vi) $x R_{k}^{\prime} y R_{k} z$

There is $a \in X_{k}$ such that $x \bowtie y \succ a \bowtie z$, where $x \in X_{k}$. Clearly, $a \succcurlyeq x$ is not possible. Since $X_{k}$ is a chain, and both $x$ and $a$ are in $X_{k}, x \succ a$. If $z \succcurlyeq x$, then $z \succ a$. It is a contradiction. Thus, either $x \succ z$ or $x \bowtie z$. Since $x \in X_{k}$, the latter implies $x R_{k}^{\prime} z$. In both cases, $x \unrhd_{k} z$. Thus, $\unrhd_{k}$ is transitive. Thus, $\unrhd_{k}$ is a partial order.

Step 2: $\unrhd_{k}$ is weakly separable.

Since $\succcurlyeq$ is lower separable, there is a countable set $Y$ in $X$ which is both $\succcurlyeq$-dense and lower $\succcurlyeq$-dense. We claim that $Y$ is $\unrhd_{k}$-dense in $X$. Let $x, y \in X$ such that $x \triangleright_{k} y$, where $\triangleright_{k}$ is the strict part of $\unrhd_{k}$.

i) $x \succ y$

Since $Y$ is $\succcurlyeq$-dense, there is $z \in Y$ such that $x \succ z \succ y$. Thus, there is $z \in Y$ such that $x \triangleright_{k} z \triangleright_{k} y$.

ii) $x R_{k}^{\prime} y$

By definition of $R_{k}^{\prime}, x \in X_{k}$ and $x \bowtie y$. By lower $\succcurlyeq$-denseness of $Y$, there is $z \in Y$ such that $x \bowtie z \succ y$. Since $x \in X_{k}, x R_{k}^{\prime} z$. Clearly, $x, y, z$ are all distinct. Thus, $x \triangleright_{k} z \triangleright_{k} y$.

iii) $x R_{k} y$

There is $a \in X_{k}$ such that $x \succ a \bowtie y$, where $x \notin X_{k}$. Since $Y$ is lower $\succcurlyeq$-dense, there is $z \in Y$ such that $x \succ a \rtimes z \succ y$. Then, $x R_{k} z$. Since $x$ and $z$ are distinct, $x \triangleright_{k} z \triangleright_{k} y$. We conclude that there is a countable set $Y$ in $X$, which is $\unrhd_{k}$-dense in $X$. Thus, $\unrhd_{k}$ is a weakly separable partial order on $X$.

Step 3: Constructing multi-vector utility indices that represent $\succcurlyeq$.

We have shown that $\left(X, \unrhd_{k}\right)$ is a poset and for each $k=1, \ldots, n, \unrhd_{k}$ is weakly separable. By 
Theorem 1 above, there is a function $\varphi_{k}: X \rightarrow[0,1]$ such that, for each $x, y \in X$ and for each $k$, $x \triangleright_{k} y$ implies $\varphi_{k}(x)>\varphi_{k}(y)$. Note that, for each $k, x \succ y$ implies $x \triangleright_{k} y$ and $\varphi_{k}(x)>\varphi_{k}(y)$. Suppose there are $x, y \in X$ such that $x \bowtie y$. Since $X_{1}, \ldots, X_{n}$ is a partition of $X$, and for each $k,\left.\succcurlyeq\right|_{X_{k}}$ is complete, there are $i, j$ with $i \neq j$, such that $x \in X_{i}$ and $y \in X_{j}$. By definition of $R_{k}^{\prime}$, $x R_{k}^{\prime} y$ and $y R_{k}^{\prime} x$. Thus, $x \triangleright_{i} y$ and $y \triangleright_{j} x$. Thus, $\varphi_{i}(x)>\varphi_{i}(y)$ and $\varphi_{j}(y)>\varphi_{j}(x)$. Finally, for each $x \in X$, define $\mathbf{u}(x)=\left(\varphi_{1}(x), \ldots, \varphi_{n}(x)\right)$. This completes the proof.

Our next result is on the upper semicontinuous representation of $\succcurlyeq$. This is an important result because upper semicontinuity is often sufficient for maximization of utility functions.

Theorem 4 Let $X$ be a topological space and let $\succcurlyeq$ be a near-complete and lower separable partial order on $X$ such that $\{y: x \succ y\}$ is open for each $x \in X$. Then there exists an upper semicontinuous mapping $\mathbf{u}: X \rightarrow[0,1]^{n}$ with $n$ being the width of $(X, \succcurlyeq)$, such that $x \succcurlyeq y$ if and only if $\mathbf{u}(x) \geq \mathbf{u}(y)$ for each $x, y \in X$.

Proof. Following Ok (2002), we define the mapping $u_{k}: X \rightarrow[0,1]$ as

$$
u_{k}(a)=\left\{\begin{array}{lc}
\varphi_{k}(x) & \text { if } U(a)=\emptyset \\
\operatorname{Inf}_{b \in U(a)} \operatorname{Sup}_{t \in L(b)} \varphi_{k}(t) & \text { otherwise }
\end{array}\right.
$$

where $\varphi_{k}$ is the utility function in Theorem 3, for each $a \in X, U(a)=\{b: b \succ a\}$ and $L(a)=\{t: a \succ t\}$. Let $x, y \in X$. If $x \succ y$, then, by weak separability, there is $z \in U(y)$ such that $x \succ z$. By Theorem 1 above, $\varphi_{k}(x)>\varphi_{k}(z)$. Since $U(y) \neq \emptyset$ and

$$
u_{k}(y)=\operatorname{Inf}_{b \in U(y)} \operatorname{Sup}_{t \in L(b)} \varphi_{k}(t) \leq \operatorname{Sup}_{t \in L(z)} \varphi_{k}(t)
$$

where $z \in U(y), \operatorname{Sup}_{t \in L(z)} \varphi_{k}(t) \geq u_{k}(y)$. Thus, by definition of $u_{k}$,

$$
u_{k}(x) \geq \varphi_{k}(x)>\varphi_{k}(z) \geq \operatorname{Sup}_{t \in L(z)} \varphi_{k}(t) \geq u_{k}(y) .
$$

Thus, $x \succ y$ implies that, for each $k, u_{k}(x)>u_{k}(y)$. Now, suppose $x \bowtie y$. By lower separability, there is $z \in X$ such that $x \bowtie z \succ y$. Since $X_{1}, \ldots, X_{n}$ is a partition of $X$ and each set in this 
partition is a chain, $x \in X_{k}$ and $z \notin X_{k}$ for some $k$. Thus, $x \triangleright_{k} z$ and $\varphi_{k}(x)>\varphi_{k}(z)$. Since $z \succ y$, the inequalities above apply. Thus,

$$
u_{k}(x) \geq \varphi_{k}(x)>\varphi_{k}(z) \geq \operatorname{Sup}_{t \in L(z)} \varphi_{k}(t) \geq u_{k}(y) .
$$

Since $\bowtie$ is symmetric, for some $l \neq k, u_{l}(y)>u_{l}(x)$. Thus, $x \succ(=) y$ holds if and only if, for each $k, u_{k}(x)>(=) u_{k}(y)$ Thus $\succcurlyeq$ is represented by $\mathbf{u}=\left(u_{1}, \ldots, u_{n}\right)$. Finally, we need to show that, for each $k, u_{k}$ is upper semicontinuous. To this end, we repeat the argument in the proof of Theorem 2 in Ok (2002): First, note $u_{k}^{-1}[\theta, 1]$ is closed for $\theta \leq 0$ or $\theta \geq 1$. For upper semicontinuity, let $\theta \in(0,1)$. Let $\left(x_{\alpha}\right)_{\alpha \in A}$ be a sequence in $X$ such that, for each $\alpha \in A, x_{\alpha} \rightarrow a$ and $u_{k}\left(x_{\alpha}\right) \geq \theta$. If, for some $\alpha \in A, a \succcurlyeq x_{\alpha}$, then $u_{k}(a) \geq u_{k}\left(x_{\alpha}\right) \geq \theta$. Now, assume that, for each $\alpha \in A$, either $x_{\alpha} \succ a$ or $x_{\alpha} \bowtie a$. Then, lower separability of $\succcurlyeq \operatorname{implies} U(a) \neq \emptyset$. Let $b \in U(a)$, and observe that, since $L(b)$ is open, there is $\alpha(b) \in A$ such that $x_{\alpha(b)} \in L(b)$. Thus, for each $b \in U(a), \operatorname{Sup}_{t \in L(b)} \varphi_{k}(t) \geq u_{k}\left(x_{\alpha(b)}\right) \geq \theta$. It implies

$$
u_{k}(a)=\operatorname{Inf}_{b \in U(a)} S u p_{t \in L(b)} \varphi_{k}(t) \geq \theta
$$

Thus, $u_{k}$ is upper semicontinuous. Since $k$ is chosen arbitrarily, $\mathbf{u}$ is upper semicontinuous. This completes the proof.

Ok (2002) proves a corollary of this theorem; the difference is that he assumes separability instead of lower separability. Thus, Theorem 4 extends his result to lower separable partial orders.

\section{Conclusion}

We extend some of the existing results on the multi-vector utility representation by Ok (2002) in terms of separability assumptions. In its technicality, it is restricted to near-complete preferences. We showed that a lower separable partial order is representable by means of a multi-vector utility function. We also show that a lower separable partial order can be represented by an upper semicontinuous vector-valued utility function. 


\section{REFERENCES}

Aumann, R., 1962. Utility theory without the completeness axiom. Econometrica 30, 445462.

Dilworth, R., 1950. A decomposition theorem for partially ordered sets. Annals of Mathematics 51, 161-166.

Donaldson, D., Weymark, J., 1998. A quasiordering is the intersection of orderings. Journal of Economic Theory 78, 382-387.

Duggan, J., 1999. A general extension theorem for binary relations. Journal of Economic Theory 86, 1-16.

Dushnik, B., Miller, E., 1941. Partially ordered sets. American Journal of Mathematics 63, 600-610.

Eliaz, K., Ok, E. A., 2006. Indifference or indecisiveness? Choice-theoretic foundations of incomplete preferences. Games and Economic Behavior 56, 61-86.

Hiragushi, T., 1955. On the dimension of ordered sets. Sci. Rep. Kanazawa Univ. 4, 1-20.

Mandler, M., 2005. Incomplete preferences and rational intransitivity of choice. Games and Economic Behavior 50, 255-277.

Ok, E. A., 2002. Utility representation of an incomplete preference relation, Journal of Economic Theory 104, 429-449.

Peleg, B., 1970. Utility functions for partially ordered topological spaces. Econometrica 38, 93-96.

Rader, T., 1963. The existence of a utility function to represent preferences. Review of Economic Studies 30, 229-232.

Richter, M., 1966. Revealed preference theory. Econometrica 34, 635-645.

Sondermann, D., 1980. Utility representation for partial orders. Journal of Economic Theory $23,183-188$.

Szpilrajn, E., 1930. Sur l'extension de l'orde partiel. Fundamenta Mathematicae 16, 386-389. 
Trotter, W., 1975. Inequalities in dimension theory for posets, Proceedings of the American Mathematical Society 47, 311-316. 